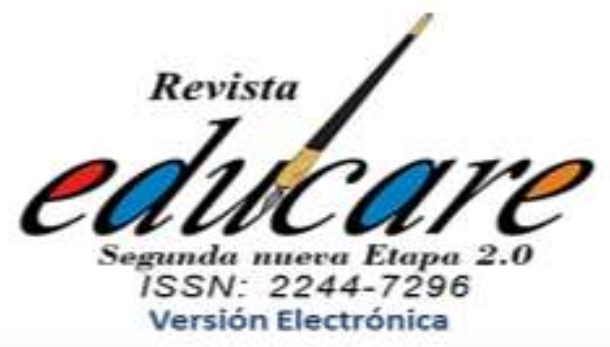

Volumen $25 \mathrm{~N}^{\circ} 1$ Enero - Abril 2021

(334-355)

\title{
JUEGOTECAS INTEGRALES: ESPACIO DE ENCUENTRO EDUCATIVO Y RECREATIVO ENTRE PADRES E HIJOS
}

Silva Marleny del Socorro

ORCID: https://orcid.org/0000-0002-6665-5849

Politécnico Colombiano Jaime Isaza

Cadavid

(Colombia)

*Doctoranda en Humanidades y Artes con mención en Ciencias de la Educación, Universidad Nacional de Rosario, Argentina. Magister en Educación: Orientación y Consejería Universidad de Antioquia. Docente investigadora del Politécnico Colombiano Jaime Isaza Cadavid. Coordinadora del Semillero de Lúdica y Saber, grupo de investigación COMAEF. dorissalazar@elpoli.edu.co

**Licenciada en Educación Preescolar y Promoción de la Familia. Especialista en Computación para la docencia, Universidad Antonio Nariño. Docente Expresiones Lúdicas en Preescolar del Politécnico Colombiano Jaime Isaza Cadavid. marlenysilva@elpoli.edu.co

\section{Recibido:}

15-10-2020

Aceptado:

30-03-2021

\author{
INTEGRAL PLAY LIBRARIES: \\ EDUCATIONAL AND RECREATIONAL MEETING \\ SPACE BETWEEN PARENTS AND CHILDREN
}



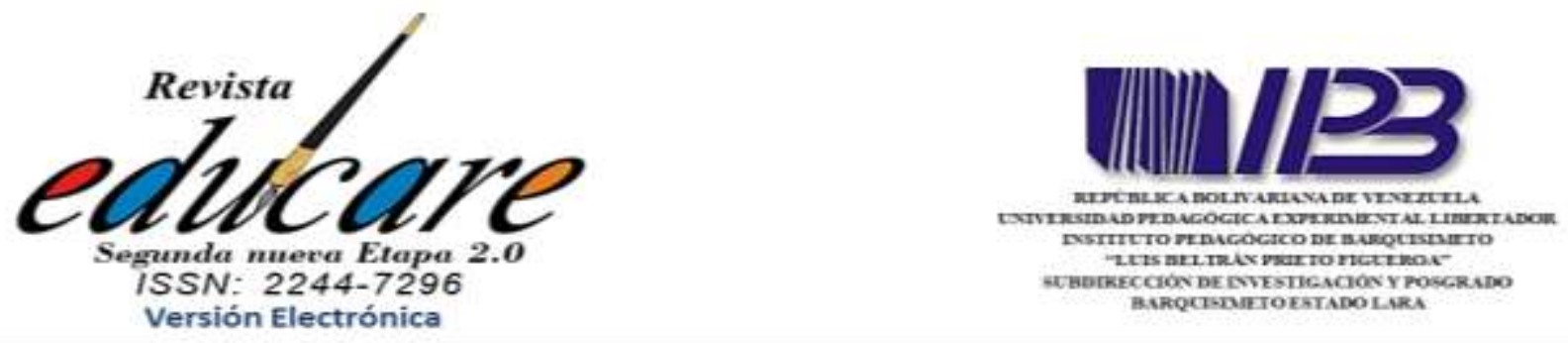

\title{
Resumen
}

Las juegotecas integrales: espacio de encuentro educativo y recreativo entre padres e hijos, constituye una propuesta educativa que surge de un proyecto de extensión solidaria, implementado con la comunidad de la escuela de iniciación deportiva del Politécnico Colombiano Jaime Isaza Cadavid. Se desarrolló una investigación social, con enfoque cualitativo de nivel descriptivo. Con esta propuesta se espera que se propicien espacios educativos y recreativos entre padres e hijos para encontrarse y jugar, posibilitar el protagonismo del niño y que sus padres reconozcan la importancia de compartir espacios de juego con sus hijos. La juegoteca representa un escenario socio cultural y educativo donde la vivencia del juego le da sentido a las relaciones interpersonales, las cuales se experimentan desde, lo hecho, lo sentido y lo expresado. Tanto el niño como el adulto podrán encontrar formas de expresar sus sentimientos y emociones, favoreciendo su crecimiento personal y fortaleciendo sus vínculos afectivos.

Palabras claves: juego, padre e hijo, espacio de encuentro.

\begin{abstract}
Integral play libraries: educational and recreational meeting space between parents and children, is an educational proposal that arises from a solidarity extension project, implemented with the community of the sports initiation school of the Polytechnic Colombiano Jaime Isaza Cadavid. Social research was developed, with a qualitative approach of a descriptive level. This proposal is expected to promote educational and recreational spaces between parents and children to meet and play, to enable the child's prominence and for their parents to recognize the importance of sharing play spaces with their children. The game library represents a socio-cultural and educational scenario where the experience of the game gives meaning to interpersonal relationships, which are experienced from, the fact, the sense and the expressed. Both the child and the adult will be able to find ways to express their feelings and emotions, promoting their personal growth and strengthening their affective bonds.
\end{abstract}

Keywords: play, father and son, meeting space 


\section{Introducción}

El presente trabajo hace parte de los resultados parciales del Proyecto de extensión solidaria de juegotecas integrales para padres e hijos del Politécnico Colombiano Jaime Isaza Cadavid. La propuesta se está implementando desde Investiguemos y Juguemos Semillero de Lúdica y Saber, el cual pertenece al grupo de investigación COMAEFI (Comunidad de Aprendizaje en Educación Física). La propuesta educativa se articuló con la vicerrectoría de extensión de la institución, con la escuela de iniciación deportiva del Politécnico y con la Licenciatura en Educación Física, Recreación y Deportes, desde dos asignaturas del plan de estudios, específicamente: expresiones lúdicas en preescolar y expresiones lúdicas en primaria, del área de lúdica, lo que favorece desde un trabajo articulado en red, impactar positivamente a la comunidad educativa.

Esta propuesta educativa, tiene como objetivos: Dar a conocer a la juegoteca como espacio educativo y recreativo entre padres e hijos para la recreación; diseñar espacios lúdicos de encuentro partiendo de las experiencias con juegos tradicionales para los padres de familia y de los juegos actuales para niños; y compartir con la comunidad politécnica los saberes y experiencias lúdicas, todo ello propuestos por los estudiantes de la licenciatura en educación física, recreación y deportes del Politécnico.

La propuesta posibilita el cumplimiento del mandato del derecho del niño a jugar, permite que este sea el protagonista y sus padres reconozcan la importancia de propiciar y compartir espacios de encuentro educativo y recreativo. Se espera que, con ésta, el adulto encuentre diferentes formas de expresar sus sentimientos y emociones sirviéndose de la juegoteca y las propuestas lúdicas que esta le ofrecen para su crecimiento personal y que sea un espacio de encuentro que fortalezca el vínculo afectivo entre padres e hijos.

De acuerdo con Dinello (2007), la actividad lúdica en una juegoteca reúne a varias generaciones, busca el crecimiento de los participantes y favorece la identificación cultural y la formación de valores sociales. Entre tanto Beatriz Caba (2012), como uno de los principales referentes de las juegotecas integrales, las define como un espacio diseñado para el juego del ser humano de todas las edades, donde intervienen un facilitador y los participantes como protagonistas del juego, agrega que "es un espacio socio cultural y educativo donde el juego le da sentido a las relaciones interpersonales e intrapersonales que 
se generan a través del afecto, la acción y las palabras" (p.199). Desde esta propuesta se estima que las juegotecas integrales son en sí misma un dispositivo de formación, diseñados para que los niños y sus padres dispongan de un espacio, tiempo, materiales y orientaciones para aprender, descubrir y jugar.

\section{Argumentación teórica}

\section{El juego}

En las políticas públicas nacionales de Colombia, en especial en lo que reza el texto del Código de la Infancia y la Adolescencia (2006) se deja claro que el juego es reconocido como un derecho fundamental de los niños. Esta orden, de naturaleza jurídica y gran espectro social requiere ser materializada, pues un decreto o una ley en sí misma no transforman, ni garantiza su cumplimiento por lo que se deben crear vías y maneras de concretarlas en el hecho. Una de las tantas formas que aporta con el cumplimiento de este derecho son los dispositivos de las juegotecas, en las cuales la actividad lúdica no se reduce a pasatiempo y distracción, sino que cobra valor y es pensada como posibilitador de constitución de identidades y construcción de aprendizajes.

En este mismo orden de ideas, el Ministerio de Educación Nacional de Colombia (2014) expone un interesante pronunciamiento donde consideran el juego;

Como derecho garantizado, invita a comprender que el niño vive en el juego y para el juego, y en esta medida se genera una actitud crítica y reflexiva frente a los espacios en los que crecen y sus condiciones; el juego merece un lugar privilegiado (p. 16)

Especialistas como Solter (2013), sostienen que el juego tiene un papel fundamental en el desarrollo integral de los niños. La pedagogía moderna recurre a él con fines educativos, ya que es un elemento de motivación que hace más agradable y facilita el aprendizaje. Se ha de entender que en el juego el niño aprende deleitándose; máxima que se apoya tras descubrir que los niños aprenden mejor y más rápidamente si lo hacen de una forma divertida y sin estrés.

A entender de Guardia y Kuiyan (2017), el juego es generador de sentido y a la vez promueve la pertenencia y el desarrollo de la identidad, tanto individual como colectiva. Para los niños el juego es una actividad seria y cuando se sumergen en él, se involucran completamente. Con la realización de actividades lúdicas, los niños exploran, experimentan 
e interactúan con el entorno, descubren la realidad, estructuran su conocimiento del mundo y reorganizan este conocimiento a la luz de los nuevos descubrimientos. En atención a esto, Aucouturier (2018) afirma que:

Un principio esencial del aprendizaje a través del juego es el de aunar las distintas esferas de la vida del niño -el hogar, la escuela, la comunidad y el mundo en general- de modo que exista una continuidad y una conectividad del aprendizaje en el tiempo y entre las diferentes situaciones. Los adultos que intervienen en cada uno de esos ámbitos tienen un papel crucial a la hora de facilitar esa continuidad y conectividad del aprendizaje, reconociendo, iniciando, guiando y organizando experiencias lúdicas que favorezcan la capacidad de acción del niño (p.28)

Otra interesante premisa es la que exponen Meneses y Montero (2019), quienes sostienen que el juego provoca situaciones de interacción social y entienden que las actividades de ocio colectivo implican la existencia de relaciones entre los participantes, tales como: relaciones de confrontación, autoritarismo, subordinación, colaboración, ayuda mutua, atención a las necesidades de los demás, cooperación, entre otras, lo que le da al juego un carácter social, convirtiéndolo en un medio importante para el aprendizaje y el desarrollo personal dentro del grupo.

En estos nuevos enfoques del juego, autores como Aucouturier (Ob.cit) resaltan que los educadores se están replanteando el modo de enseñar a los niños aprovechando su enorme potencial de aprendizaje. El juego constituye una de las formas más importantes en las que los niños obtienen conocimientos y competencias esenciales. Por esta razón, las oportunidades de juego y los entornos que favorecen el juego, la exploración y el aprendizaje práctico constituyen el fundamento de los programas de educación eficaces.

\section{El juego en la juegoteca}

El juego es una actividad tan antigua como el hombre mismo, aunque su concepto, y su forma de practicarlo, varían según la cultura de los pueblos. Montero, Monge y Alvarado (2001) estiman que el ser humano juega en forma innata, producto de una experiencia placentera como resultado de un compromiso en particular, es así como el juego se convierte en un estímulo valioso mediante el cual el individuo se vuelve más hábil, perspicaz, ligero, diestro, fuerte y sobre todo alegre. En relación al contexto educativo, Lacayo y Coello (2008) consideran que los niños aprenden a crecer en una forma recreativa, por lo que el docente 
debe estar consciente de la utilidad del juego en el desarrollo de aprendizajes. Su labor se va a ver facilitada en el sentido de tener objetivos de clase, actividades estructuradas no solo de expresión libre sino de desarrollo social, emocional e intelectual.

En atención a lo refrendado, durante generaciones, crecer fue una tarea fácil: se ibas a la escuela unas horas al día, practicabas deporte y tenías alguna afición, y el resto del tiempo se jugaba. Honore (2012) expone de manera cruda cómo nuestro moderno enfoque de la infancia es todo un fracaso: nuestros hijos están más obesos, miopes, más deprimidos y más medicados que cualquier generación anterior. Usando a los niños como forma de revivir nuestra propia vida, o para compensar nuestras frustraciones personales, hemos destruido la magia y la inocencia de la niñez.

Como alternativa en las juegotecas se entiende el juego como la actividad central que favorece el desarrollo de las dimensiones del ser. A partir del juego en la juegoteca, se estimula la capacidad de crear, aprender, decidir, compartir, construir, acordar y proponer. Una característica del juego en las juegotecas es el trabajo desde la grupalidad, convirtiéndose en el lugar del otro y los otros con los que se juega.

Como antecedente a lo expuesto Dinello (1998) destaca que el juego en la juegoteca, es vivido como un mundo de aventuras lúdico-creativas, que le facilita al niño conocerse mejor a sí mismo, expresar su forma de ser, de pensar y de sentir. El juego en la juegoteca posibilita al niño que explore su mundo. Es a través de este, que el niño representa el entorno sociocultural que habita. En nuestra propuesta educativa, las juegotecas confluyen en diversas expresiones lúdicas como las artes plásticas por medio del dibujo, la pintura y las manualidades. Las teatrales a través de los títeres, las dramatizaciones y el cuento. Las expresiones lúdico-musicales como el canto, la danza, la expresión corporal y la ronda. También en las juegotecas los juegos tradicionales tienen su lugar y las demás actividades que inviten a jugar de manera espontánea.

Se hace viable considerar que el juego es indispensable para el desarrollo del ser desde todas sus dimensiones dadas sus innumerables bondades. Es un puente que permite el acercamiento y la comunicación entre los niños y los adultos produciendo beneficios, tanto en uno, como en el otro. 


\section{El derecho del niño a jugar}

El juego, además de ser un derecho del niño, es fundamental para su desarrollo integral. Desde la legislación en la Constitución política de Colombia (1991) se le ha dado un lugar primordial que busca garantizar el derecho de todos los niños a jugar. En el orbe mundial en 1959 las Naciones Unidas aprueban la Declaración de los Derechos del niño, donde establece 10 principios, entre ellos "El derecho a actividades recreativas y a una educación gratuita". (Artículo 7). En este principio el juego constituye una actividad esencial para el desarrollo del niño al mismo nivel de los otros principios que reconocen el derecho del niño a la protección, al nombre, la vivienda, la alimentación, la salud, la comprensión y al amor de los padres y de la sociedad.

Luego, la ONU, declara la Convención sobre los Derechos del Niño (1989), la cual hace referencia al juego, en los siguientes términos: "Los Estados Partes reconocen el derecho del niño al descanso, al esparcimiento, al juego y a las actividades recreativas propias de su edad y a participar libremente en la vida cultural y en las artes" (artículo 31). En este sentido el Estado debe ser garante de este derecho, y por lo tanto desde sus diversas instituciones, apoyar estrategias que garanticen su acceso y cumplimiento.

En Colombia la Constitución del 91 en el artículo 44 plantea "Son derechos fundamentales de los niños: la vida, la integridad física, la salud y la seguridad social, la alimentación equilibrada, su nombre y nacionalidad, tener una familia y no ser separados de ella, el cuidado y el amor, la educación y la cultura, la recreación y la libre expresión de su opinión", en tanto el juego como elemento propio de la recreación es reconocido como un derecho fundamental. El código de Infancia y adolescencia (Colombia, 2006) reconoce que “Los niños, las niñas y los adolescentes tienen derecho al descanso, al esparcimiento, al juego y demás actividades recreativas propias de su ciclo vital y a participar en la vida cultural y las artes" (artículo 30).

La Ley General de Educación (1994) en el artículo 5, estipula los fines de la educación, el fin 12 hace referencia a "la educación física, la recreación, el deporte y la utilización adecuada del tiempo libre”. El Decreto 2247 (1997) que contempla las orientaciones curriculares para el nivel preescolar en Colombia, comprende la lúdica como un principio que: 
Reconoce el juego como dinamizador de la vida del educando mediante el cual construye conocimientos, se encuentra consigo mismo, con el mundo físico y social, desarrolla iniciativas propias, comparte sus intereses, desarrolla habilidades de comunicación, construye y se apropia de normas. Así mismo, reconoce que el gozo, el entusiasmo, el placer de crear, recrear y de generar significados, afectos, visiones de futuro y nuevas formas de acción y convivencia, deben constituir el centro de toda acción realizada por y para el educando, en sus entornos familiar, natural, social, étnico, cultural y escolar (Artículo 11).

Desde este principio consideramos que el juego no solo debe ser reconocido en el nivel preescolar, sino que el compromiso es darle su lugar en los demás niveles de educación, ya que es un recurso de una riqueza invalorable. Desde nuestra experiencia como docentes, hemos evidenciado que en el imaginario de muchos docentes este se asocia con pérdida de tiempo. Conocemos experiencias donde en el nivel de la básica primaria es relegado a un segundo plano, sólo se privilegia en el descanso pedagógico.

\section{¿Ludotecas o juegotecas?}

Las juegotecas son entendidas por algunos autores como sinónimo de ludotecas. Desde nuestra experiencia, ambas tienen elementos comunes, pero no son lo mismo. El término ludoteca proviene del latín ludus que significa juego o juguete. La ludoteca es un lugar diseñado donde se disponen diferentes tipos de juguetes o juegos destinados a diversas poblaciones, principalmente niños.

El Instituto de Deportes y Recreación de Medellín (INDER), utiliza la estrategia de ludotecas con el fin de ofrecer espacios de juego, ocio y tiempo libre para los niños y sus familias. "Los espacios son diseñados y adaptados para el desarrollo motriz y se utilizan juegos y juguetes que promueven el encuentro para la construcción de ciudadanía" (INDER, 2020, parr. 1). Cada ludoteca establece metodologías a partir de las necesidades de la población beneficiaria. En la actualidad el programa ofrece 70 ludotecas.

La ludoteca es un dispositivo en el cual, a partir de la acción lúdica, se promueve el desarrollo de los niños como sujetos sociales, con participación activa en la construcción de los espacios y las propuestas que promueven y legitiman el juego. Las ludotecas son diseñadas de acuerdo a las características, las necesidades y las particulares del lugar, de los niños, de sus familias y de la comunidad donde se encuentra. En Argentina, desde el programa de Juegotecas Barriales se las entienden según Guardia y Kuiyan, (2017) como: 
... espacios de juego para niños de 2 a 13 años, quienes concurren en forma sistemática y voluntaria, donde desarrollan actividades lúdicas, expresivas y culturales en un contexto institucional y con un equipo interdisciplinario de facilitadores lúdicos a cargo. Se trabaja a través de la participación y de experiencias compartidas entre niños, familias y las organizaciones barriales (p.14)

Este espacio de juegotecas barriales es similar al de las ludotecas que se implementan en Medellín. Estas tienen como propósito, además de la recepción de niños en el espacio, “la puesta de lo lúdico en el espacio público, en la trama social, participando así activamente de las redes territoriales y de las actividades socioculturales de los barrios donde se encuentran insertas" (Ob.cit p.16)

Por otro lado, los referentes teóricos que soportan el término de juegoteca han sido principalmente los de Dinello (2006) y Caba (2012). Estos autores definen la juegoteca como "un espacio socio educativo y cultural donde siempre hay otro con el cual comparto. Caba (Ob.cit) le acuña al término de juegoteca, el de integral, como un "espacio diseñado especialmente para el juego del ser humano desde su más temprana edad hasta que el ser humano lo decida" (p.199). Reafirma Caba (Ob.cit) que en las juegotecas integrales "el niño es invitado a jugar y a interactuar con otros en una relación lúdica placentera en forma protagónica" (p.100).

En cuanto a su diseño, Aubert y Caba (2013) recomiendan no encasillarse en un formato preestablecido, sino construirlas de forma grupal teniendo en cuenta las necesidades e intereses de la población. Proponen diseñar las juegotecas desde las ocho inteligencias múltiples de Gardner y Nogués (1995) a saber: lógica matemática, lingüística verbal, interpersonal, visual, espacial, musical, corporal cinestésica, intrapersonal y naturalista. En cada espacio lúdico se disponen materiales e instructivos para que los participantes se dispongan a jugar.

Desde la presente propuesta educativa, cuando se nombran las ludotecas, se hace referencia a las institucionalizadas, con salas establecidas, ya sean hospitalarias, barriales o comunales y cuando se referencia a las juegotecas, se trata del espacio que se crea y se diseña aprovechando cualquier espacio adecuado y seguro con el fin de invitar a los niños y a sus familias a jugar. En la tabla 1 se identifican diferencias y similitudes entre las ludotecas y la propuesta de juegotecas integrales que se lleva a cabo en el Politécnico. 
Se puede destacar como elementos comunes de ambos dispositivos de juego que son pensados para responder a la necesidad de legitimar el derecho del niño a jugar, establecen metodologías a partir de las necesidades de la población. En ambos dispositivos el niño es el protagonista al mismo tiempo el juego se reconoce como medio de expresión de la cultura y en estos espacios siempre hay otro con quien jugar.

Tabla 1

Diferencias y similitudes entre las ludotecas y el proyecto de juegotecas

\begin{tabular}{lll}
\hline & Ludotecas & $\begin{array}{l}\text { Proyecto educativo juegotecas } \\
\text { integrales }\end{array}$ \\
\hline $\begin{array}{l}\text { Lugar donde se lleva a } \\
\text { cabo }\end{array}$ & Fijo, preestablecido & Acondicionado cada 8 días \\
Materiales dispuestos & $\begin{array}{l}\text { Juegos, juguetes y } \\
\text { alternativas } \\
\text { pedagógicas }\end{array}$ & $\begin{array}{l}\text { Dependiendo de las actividades } \\
\text { a desarrollar se prefiere que } \\
\text { sean reciclables, del medio y } \\
\text { material didáctico, }\end{array}$ \\
& $\begin{array}{l}\text { Motriz } \\
\text { Componente central }\end{array}$ & $\begin{array}{l}\text { Inteligencias múltiples y } \\
\text { dimensiones del desarrollo }\end{array}$ \\
& $\begin{array}{l}\text { Salas o centros de } \\
\text { interés }\end{array}$ & Rincones o espacios lúdicos \\
Espacios & Silva (2019) &
\end{tabular}

Adaptado por Salazar y Silva (2019)

\section{Descripción de la propuesta}

La propuesta educativa nace de una idea de investigación formativa. Cuando ya se había formulado e inscrito en la dirección de investigación del Politécnico, el equipo investigador consideró la necesidad de apropiarse más de este dispositivo lúdico, para comprenderlo y hacer innovaciones. Se analizó la viabilidad de integrar esta temática al currículo de las asignaturas expresiones lúdicas en preescolar y expresiones lúdicas en primaria, desde Investiguemos y Juguemos Semillero de Lúdica y Saber. Se presentó el proyecto al Consejo de Facultad de la Licenciatura, quien recomendó la articulación con la escuela de iniciación deportiva, para favorecer a la población que asiste los sábados a las diferentes actividades lúdicas y deportivas en la institución. Posteriormente, el proyecto se presentó a la vicerrectoría de extensión de la institución como un proyecto de extensión 
solidaria el cual la avaló y recomendó ampliar la invitación a participar del programa a toda la comunidad politécnica, lo que posibilitó ampliar la cobertura beneficiaria.

Desde esta propuesta educativa comprendemos las juegotecas integrales como dispositivos de formación, diseñados para que los niños y sus padres o cuidadores dispongan de espacio, tiempo, materiales y orientaciones para jugar. El jugar es una actividad fundante y primordial en la infancia. Las experiencias lúdicas por las que transita todo niño, aportan a su crecimiento, a su desarrollo y a sus modos de establecer vínculos con los que lo rodean. A través de su práctica el niño incorpora normas, valores y costumbres propios del contexto en el que se desarrolla. Jugar genera sentido, promueve la pertenencia y el desarrollo de la identidad, tanto individual como colectiva.

Al jugar, los niños se comprometen, toman una posición activa, imaginan, inventan, crean, conocen y actúan explorando con todos sus sentidos. Por otra parte, se ha de reconocer que en muchos casos el contexto donde la mayoría de niños no tienen la posibilidad de jugar en su misma casa, por ello se necesita rescatar lo lúdico y brindarle un lugar privilegiado en la infancia promoviendo tanto el juego aplicado con fines didácticos, como la necesidad de que los niños jueguen por el solo valor de jugar.

En este dispositivo el juego cobra valor y se legitima como posibilidad de encuentro con el otro y con los otros desde un enfoque grupal, no competitivo. En las juegotecas integrales se tienen en cuenta los intereses y necesidades del niño como ejes de acción, la integración de los padres o cuidadores para que reconozcan en el juego la posibilidad de encuentro con su hijo y el fortalecimiento de las relaciones interpersonales con todos los participantes. La propuesta se implementa con un grupo conformado por tres docentes del área de lúdica, cuatro estudiantes en formación que están haciendo su trabajo de grado desde este proyecto articulados con el Semillero de Lúdica y Saber y tres estudiantes de la Universidad colombiana Eafit quienes se vincularon a este proyecto social.

nicialmente por medio de un curso teórico práctico se capacita en el dispositivo lúdico de juegotecas a los estudiantes que cursan las asignaturas de expresiones lúdicas en preescolar y expresiones lúdicas en primaria (aproximadamente noventa estudiantes cada semestre). Se trabaja la fundamentación legal del derecho del niño a jugar y los elementos teóricos de la juegoteca. Se vivencia una juegoteca en la que los estudiantes son los protagonistas. Cada espacio lúdico se diseña de tal forma que se motiven con los diversos 
materiales y los instructivos que encuentran allí, los cuales proponen retos y propician el desarrollo de la creatividad, de la libre expresión y del trabajo grupal. Se privilegian los elementos del trabajo cooperativo.

Vivir esta experiencia y participar en la capacitación son fundamentales, ya que en la metodología que implementamos como docentes orientadoras (facilitadoras del juego) diseñamos una juegoteca con todos sus elementos (saludo, actividad grupal, conteos, experiencia en los espacios lúdicos, momento de reflexión, registro fotográfico y despedida). Durante la capacitación invitamos a los estudiantes a que participen de cada uno de los espacios lúdicos, ellos disfrutan cada uno de los momentos de la juegoteca, lo que posibilita la sensibilización y apropiación de este dispositivo. Los estudiantes manifiestan que el comprender las dinámicas que se viven en la juegoteca, desde la experiencia, siendo ellos los protagonistas, contribuye a que sean más creativos e innovadores en su diseño e implementación.

Para el desarrollo de las juegotecas, las cuales son consideradas dentro del currículo de la licenciatura como microprácticas, los estudiantes se organizan en grupos cooperativos, en el que cada uno asume un rol (líder, comunicador, utilero, relator y vigía del tiempo). Cada sábado hacen la intervención dos grupos cooperativos, uno con la población de niños en edad preescolar en el horario de 8:00 am a 10:00 am y el otro con los niños en edad escolar en el horario de 10:00 am a 12:00 m. Se atiende a un grupo poblacional de diferente disciplina deportiva: futbol, baloncesto, taekwondo, gimnasia, natación, patinaje, bádminton y voleibol, los cuales, junto con sus profesores, sus padres y cuidadores, asisten a la juegoteca como un espacio prediseñado por los estudiantes que realizan su micropráctica. La juegoteca se presenta con actividades planeadas las cuales, aunque pueden modificarse, respetan cierta estructura que le da identidad.

Al llegar los niños y sus familias al espacio diseñado para la juegoteca, los estudiantes practicantes los saludan por medio de cantos, seguidamente realizan una actividad grupal que generalmente es una ronda, luego a través de conteos realizan la división grupal y se invita a participar a todos los asistentes de los espacios lúdicos.

Para cada espacio se determinan unos tiempos, pero en caso de que algún niño se quiera quedar en uno por más tiempo se le permite. Para finalizar se disponen en círculo todos los participantes y se hace una actividad de evaluación en la que circula la palabra de 
los niños, de sus padres y de sus profesores. Esto implica un momento de reflexión que posibilita recuperar sus voces como parte del proceso y favorece la retroalimentación con las observaciones realizadas por los estudiantes practicantes. Todo esto sirve como insumo para planear nuevos encuentros. Para el cierre, se realiza una actividad grupal de despedida y se invita a los participantes para hacer un registro fotográfico.

Los niños y sus familias transitan en las juegotecas el placer de lo lúdico. Es un espacio donde pueden desarrollar su autonomía, encontrar compañeros de juego, proponer reglas, acuerdos, solucionar situaciones y especialmente tomar decisiones frente a la forma de jugar, de construir, de crear, de aprender y de imaginar.

Para el diseño de las juegotecas se tiene un formato en el que los estudiantes elaboran la planeación que les corresponde. Durante el primer el semestre (2019-1) se planearon e implementaron desde la propuesta de Caba (2012) quien las propone para fortalecer el desarrollo de las inteligencias múltiples. Esta experiencia inicial con las juegotecas integrales, nos llevó a hacer ajustes e innovaciones para la población en edad preescolar. Surge entonces la idea de pensar las juegotecas teniendo en cuenta los lineamientos curriculares del decreto 2247 (Colombia, 1997, MEN), el cual establece las normas para la prestación del servicio preescolar en Colombia y plantea el trabajo por dimensiones del desarrollo del ser.

Se decidió para el semestre 02 de 2019 capacitar a los estudiantes que cursan la asignatura expresiones lúdicas en preescolar en juegotecas teniendo en cuenta las características del niño en esta edad y las dimensiones del desarrollo humano. El trabajo inicial, permitió desde la evaluación y la reflexión permanente de los diferentes actores, asumir retos y desafíos con los niños en edad preescolar y buscar otras formas de diseñar los dispositivos lúdicos de las juegotecas teniendo en cuenta las características y las particularidades de los niños, las formas de comunicarse y de jugar.

Es así como se pensó la juegoteca con siete bases, una para cada dimensión del desarrollo (corporal, cognitiva, socio afectiva, ética, estética, espiritual y comunicativa) e instructivos cortos seguidos de la expresión "somos": músicos, constructores, felices, amigos, artistas, paz, actores, los cuales invitan a jugar de manera grupal utilizando los diversos materiales encontrados en cada una de las bases lúdicas. 
A continuación se presenta en la tabla 2 el cuadro que sirvió de referente a los estudiantes para la planeación semanal de juegotecas a implementar con los niños en edad preescolar desde las dimensiones del desarrollo a partir de esta experiencia, con ello se ordeno, siguió y evaluó de manera sistematizada el trayecto de experiencia con las juegotecas.

Tabla 2

Instructivos para rincones lúdicos desde las dimensiones del desarrollo.

\begin{tabular}{lll}
\hline $\begin{array}{c}\text { Dimensiones del } \\
\text { desarrollo }\end{array}$ & \multicolumn{1}{c}{ Instructivos } & \multicolumn{1}{c}{ Materiales } \\
\hline Corporal & Somos músicos & $\begin{array}{l}\text { Instrumentos musicales:maracas, tambores, flautas, guitarras, } \\
\text { panderetas, cascabeles, pelucas, gafas. }\end{array}$ \\
Cognitiva & $\begin{array}{l}\text { Somos } \\
\text { constructores }\end{array}$ & Tapas de colores, arma todo, bloques de madera, regletas \\
Socioafectiva & Somos felices & Juguetes y elementos de la infancia traídos por los estudiantes. \\
Ética & Somos amigos & Bombas infladas, marcadores y recreolona (paracaídas) \\
Estética & Somos artistas & Papel globo, tijeras, cinta, colbón, papel periódico, pinturas. \\
Espiritual & Somos paz & Pebetero, esencias, tapete, cojines, música, parlante \\
Comunicativa & Somos actores & Disfraces, telas, collares, gafas, pelucas, maquillaje.
\end{tabular}

Salazar y Silva (2019)

Una de las docentes del proyecto, desde su formación como licenciada en educación física, se propuso, con su grupo, diseñar juegotecas para fortalecer el desarrollo de habilidades motrices de los niños, lo cual se convirtió en una innovación sin perder el espíritu de la juegoteca. La evaluación de cada uno de los encuentros permitió darnos cuenta de lo significativo que era para los niños de la disciplina deportiva de natación el elemento agua y para los de patinaje los patines. Situación que propició que nos diéramos a la tarea de diseñar juegotecas teniendo en cuenta los escenarios deportivos, los elementos propios de cada deporte y las orientaciones dadas por los profesores entrenadores de los niños en estas disciplinas.

El instrumento para la planeación (Tabla 3) es un formato que contiene; objetivo de la juegoteca, población beneficiaria, duración de la intervención, materiales y cantidad, descripción de los momentos. En cuanto a las actividades destacan recibimiento; saludo, rompe-hielo y división de grupo. Actividad central; diseño de cada uno de los espacios 
lúdicos con sus instructivos. Actividad final; evaluación del encuentro y actividad grupal de despedida.

Tabla 3

Instrumento de planeación juegotecas integrales

Formato para la planeación de las juegotecas integrales

Nombre de la actividad:

Población beneficiaria:

Lugar:

Fecha: Hora:

Objetivo:

Momentos

Actividades

Dimensión

Materiales

Evaluación

del /cantidad

Recibimiento y saludo

desarrollo

Central

Cierre

Evaluación

Actividad grupal de

despedida

Salazar y Silva (2019)

Entre tanto, el docente, les hace ajustes y recomendaciones a los estudiantes a partir de

la planeación propuesta. Otro instrumento que se diseñó con el fin de sistematizar el proyecto

fue un formato de entrevista a padres o cuidadores de los niños, con las siguientes preguntas

(Tabla 4)

Tabla 4

Formato de entrevista para padres o cuidadores

Instrumento de entrevista para padres o cuidadores

- ¿Cuáles son los juegos preferido por el niño?

- ¿Cuáles son los juguetes preferidos por el niño?

- ¿Cuáles fueron los juegos preferidos por usted cuando tenía la edad de su hijo?

- ¿Cuáles fueron los juguetes preferidos por usted cuando tenía la edad de su hijo

- ¿A qué juega con su hijo?

- ¿Con que frecuencia juega con su hijo?

- En caso de que tuviese más tiempo para compartir con su hijo ¿a qué le gustaría jugar y por qué?

Estudiante entrevistador:

Fecha:

Salazar y Silva (2019).

La recolección de los datos obtenidos en las entrevistas a los padres o cuidadores se realiza semanalmente, los estudiantes en formación cumplen con este compromiso. Estas respuestas sirven de insumo para planear las siguientes juegotecas y tener más elementos 
para involucrar a los padres y acudientes en el programa. Adicionalmente, se diseñó un instrumento en el que por grupos cooperativos los estudiantes presentan un informe de la evaluación de la experiencia, el cual contiene: descripción de la juegoteca, aprendizajes adquiridos, aspectos a mejorar, registros fotográficos y/o pequeños videos.

Las experiencias se socializan en el siguiente encuentro académico, lo que posibilita un espacio de reflexión por parte de los estudiantes y tener en cuenta las observaciones y recomendaciones para nutrir las siguientes microprácticas y hacer los ajustes y adaptaciones necesarias.

\section{¿Y en la época de pandemia qué? juegotecas en casa}

La época de confinamiento debido a la pandemia por el covid 19, la vimos como una oportunidad para pensar en alternativas de continuar con la propuesta educativa desde casa. Se trata de disponer los espacios de la casa para que el niño los habite desde las experiencias lúdicas. Como primera opción diseñar en un espacio de la casa (garaje, patio) una juegoteca compuesta por varios rincones lúdicos con materiales que inviten al niño a jugar. Como por ejemplo un rincón de construcción, uno de preparación y consumo de alimentos, otro de juego roles, otro de juegos de mesa, otro de actividades plásticas, otro de juegos predeportivos y los demás de acuerdo con los intereses del niño, a las iniciativas y materiales con que se cuente en el hogar.

La segunda alternativa se trata de habilitar cada uno de los espacios de la casa para que el niño los apropie para jugar. Pensar por ejemplo en el cuarto del niño como el espacio que le pertenece y en el que puede jugar de manera libre utilizando cada rincón, al tiempo que se pueden desarrollar proyectos como el de lavar los juguetes y los materiales de juego, organizarlos, clasificarlos en cajas que puede decorar el niño como una actividad plástica. El comedor puede ser el espacio que convoque a los juegos de mesa, a armar un rompecabezas y a juegos con lápiz y papel. La sala el lugar donde se puede armar una carpa o un cambuche para dormir, el espacio de estudio estar dispuesto con diversos materiales que convoquen a las actividades lúdicas plásticas y a las manualidades y la cocina como espacio para preparar recetas con la compañía y orientación de los padres o de un adulto.

\section{A manera de cierre: reflexiones finales y recomendaciones}

El dispositivo de juegotecas integrales, propuesto por Caba (2012) que pretende 
estimular las inteligencias múltiples, sirvió de fundamento e inspiración para la propuesta educativa de "Juegotecas integrales: espacio de encuentro educativo y recreativo entre padres e hijos" Desde la experiencia se reconoció la necesidad de salir de la zona de confort y pensar en otras maneras de diseñar propuestas divergentes desarrollando las competencias propositivas. Se puede identificar como valor agregado de la propuesta, el elemento de transformación, en cuanto a las formas de implementar las juegotecas, de sus temáticas y de sus ejes de acción. Consideramos que el elemento que motivó estas transformaciones han sido los momentos de reflexión que posibilitan comprender que las prácticas reflexivas no se limitan a ejercicios procedimentales de repetición, de réplica, de imitación, que es necesario pensarlas, repensarlas y adecuarlas a las características de la población que se interviene y a los recursos con que se cuenta. Al respecto Perrenoud (2007) afirma que,

Solamente un formador reflexivo puede formar a enseñantes reflexivos, no solamente porque encarna globalmente lo que preconiza sino porque aplica la reflexión «de forma espontánea», en el curso de una pregunta, una discusión, una tarea o un fragmento de saber (p.70)

Las juegotecas diseñadas en el marco de esta propuesta tiene el potencial de convertirse en referentes para ser implementadas en otros contextos, incluso en la casa. Conscientes de la necesidad de legitimar el derecho del niño a jugar y de la preocupación de que en esta época gran parte del tiempo se la pasen frente a los dispositivos electrónicos, se propone las juegotecas como dispositivo lúdico para jugar en casa, experiencia que se diseñó y actualmente se está socializando en eventos académicos con el fin de que los padres se sirvan de ella en esta época de confinamiento por el covid 19. Al respecto Morin (2016) considera que "Es necesario que todos los que tienen la misión de enseñar ocupen la vanguardia de la incertidumbre de nuestro tiempo" (p.43)

El trabajo riguroso por parte del equipo de docentes investigadores de la propuesta educativa de Juegotecas integrales: espacio de encuentro educativo y recreativo entre padres e hijos le ha otorgado un marco de referencia que permite comprenderlo y diferenciarlo de otros dispositivos lúdicos como el de las ludotecas y de otros tipos de eventos recreativos que también convocan a los niños a jugar y que tienen otras o similares intencionalidades. Desde esta mirada las juegotecas han ganado espacio a tal punto de ser reconocida como un 
programa a nivel institucional que garantiza el derecho del niño a jugar al tiempo que ha servido de referente para ser implementada en otros escenarios educativos y recreativos.

El juego en las juegotecas es comprendido como un derecho del niño, al mismo nivel que los otros reconocidos por la Declaración de los derechos del niño. Desde el contexto educativo escolar se le otorga el espacio al juego en el descanso pedagógico (recreo). Esta apreciación converge con la afirmación de Pavia (2012) en relación a que "los patios de recreo interesan desde la perspectiva del derecho al juego. "Los fugaces momentos de recreos en la escuela constituyen una actividad de alta valoración en el marco de su vida cotidiana" (p.11). Los aportes de Valdiño (2015) sobre el juego en la escuela primaria permiten confirmar que esta le sigue dando prioridad a la selección y ampliación de contenidos curriculares, desconociendo el valor incalculable del juego, justificándose desde el discurso, en el interés por el aprendizaje de los alumnos.

Se diferencian en la escuela claramente dos tiempos; el del estudio y el del recreo, este último que por fortuna se da en el espacio del patio, donde desde un encuentro con los otros, hay movimiento, despliegue y juegos. Los diseños de los planes de estudio y la intensidad horaria siguen poniendo el foco en las asignaturas que son tenidas en cuenta a la hora de ranquear las instituciones a nivel nacional e internacional. En este sentido priorizan la faceta productiva de la educación, desentendiéndose de lo lúdico en los espacios de formación.

Uno de los aportes importantes de esta propuesta de Juegotecas integrales: espacio de encuentro educativo y recreativo entre padres e hijos, es que posibilitó el reconocimiento del juego como medio que facilita el fortalecimiento del vínculo afectivo del niño con sus padres, sus cuidadores, sus abuelos, su familia y con otros adultos significativos.

Las juegotecas son una fuente inagotable que favorece en el niño a través del juego el encuentro consigo mismo, con el otro y con el mundo que le rodea. En este sentido, se sugiere a partir de esta propuesta, a los docentes de educación física, recreación y deporte, de preescolar, de la básica primaria y a los agentes educativos, que apropien la juegoteca como dispositivo en sus prácticas cotidianas, como medio para legitimar el derecho del niño a jugar, mientras disfruta de experiencias de juego independientemente, que sean pensadas o diseñadas desde las inteligencias múltiples, las dimensiones del desarrollo, las habilidades 
motrices o las expresiones artísticaso del entorno en el que se vaya a desarrollar, ya sea educativo, de salud, público o del hogar.

Es requisito el propiciar espacios de juego, tiempo y recursos, tales como las juegotecas, posibilitar el cumplimiento del mandato del derecho del niño a jugar, permite que este sea el protagonista del juego, comparta, cree e imagine en compañía de sus padres. Consideramos que el juego, en la juegoteca, es primordial para el desarrollo de las dimensiones del ser y la construcción de una mejor sociedad. El juego le permite al niño conocer el mundo que lo rodea y es uno de los medios más apropiado para que establezca nuevas relaciones y encuentre solución a los problemas que percibe en su entorno.

Los agentes educativos, los padres, los educadores son los garantes de que el derecho del niño a jugar se materialice. El juego, así sea gratuito, tiene un lugar muy discreto en las propuestas de proyectos socio-educativos. Solamente aquellos adultos sensibles que están cerca del niño le dan el valor protagónico, ya que conciben el mundo del niño alrededor del juego. No es suficiente preguntarse sobre la importancia del juego en el desarrollo del niño, es necesario revisar la actitud ante lo lúdico y lo creativo y la validez que le damos a las estrategias lúdica sutilizadas en la formación de los niños.

Por ello se entiende que una de las grandes tareas que se deben plantear los especialistas en el área es el reforzamiento de elementos de valor que consoliden una conciencia social sobre el juego y su incidencia en la actividad del niño. Se ha de considerar como requisito necesario para que las juegotecas favorezcan el desarrollo integral del niño y de la comunidad, que su implementación sea a partir de las necesidades de carácter físico, social e intelectual de los niños, sus hermanos, sus padres y demás miembros de la comunidad donde se desarrollan. Sólo de la reflexión conjunta acerca de sus características y de sus particularidades se podrán brindar alternativas acordes a las necesidades de la población.

Pensar las juegotecas como alternativa lúdica en el contexto de la escuela primaria, en relación con el lugar que ocupa y podría ocupar el juego, que hoy sólo está presente en el recreo, puede contribuir a reposicionar e iluminar este y el espacio áulico de formación que, para muchos, ve interpelado su sentido social. Por ello se estima que, la juegoteca como dispositivo lúdico permite recuperar el juego y la actividad creadora en el niño. Es una alternativa para aquellos seres humanos que disfrutan poco de este tipo de espacios de juego. Creemos que la revaluación, recuperación e implementación de las actividades lúdicas, 
artísticas y en fin, de todas aquellas formas de expresión gratuita y ancestral, que se encuentran en el ser humano, son una de las posibilidades más interesantes que podemos ofrecer a la niñez. Las juegotecas desde el encuentro con los padres y los abuelos, posibilita la recuperación de los juegos tradicionales, los cuales desde esta experiencia son recibidos con alegría y asombro por parte de los niños.

Es recomendable pensar en las formas de proteger el juego como actividad espontánea, y replantearse el tiempo y los espacios escolares, ya que en la mayoría de los casos los niños están sobrecargados de actividades académicas que le apuntan a los contenidos curriculares. Por un lado, se le da valor al juego por la notable función social que cumple como fenómeno cultural. Y por el otro, se le concibe como opuesto al trabajo, como pérdida de tiempo, actividad no seria, que no persigue fines utilitarios. La escuela es la institución encargada de rescatar nuevamente al juego desde su configuración socio cultural para dar respuesta a la necesidad del niño a jugar como actividad fundamental en la vida escolar, desde el rescate y el diseño de espacios lúdicos necesarios para que el niño disfrute y se desarrolle de forma armónica.

Se insiste en que el juego debe ser garantizado en todos los entornos del niño, en el hogar, en el educativo, en el barrial, en el hospitalario. La juegoteca es un dispositivo versátil que cabe en todos estos espacios y lo garantiza. El juego, en la juegoteca, permite al niño desarrollar todo su potencial en actividades creativas, reflexivas y lúdicas, ya sean individuales o colectivas, por medio de las cuales el niño puede expresar libremente su yo, comunicarse, llegar a acuerdos, tomar decisiones y resolver problemas.

Se hará empeño en seguir investigando y asumir el reto de apropiar este dispositivo para hacer innovaciones educativas en el campo de otras áreas como la biología, la física, las matemáticas y las ciencias naturales. Además de utilizar este dispositivo lúdico con otro tipo de poblaciones como la del adulto mayor o el universitario.

\section{Bibliografía}

Aucouturier, B. (2018) Actuar, jugar, pensar. Puntos de apoyo para la práctica psicomotriz educativa y terapéutica. Grao.

Caba y Aubert. (2013) Repertorio lúdico, infancia y escuela: pensando en términos del derecho del niño a jugar. En Sarlé P (pp 89-103). Buenos Aires: Homo Sapiens. 
Caba, B (2012) Juegoteca Integral: la evolución de un concepto con corazón. En Primer

Congreso Internacional. Neuropedagogía Lúdica. Fundación Ecosófica de Colombia. Bogotá, Colombia.

Colombia. Ministerio de Educación Nacional (MEN), Camargo, A. M., Martín, C. M. C., Duran, S. M., Reyes, Y., \& Chaves, V. (2014). El juego en la educación inicial. Colombia: Ministerio de Educación.

De Colombia, C. P. (1991). Constitución política de Colombia. Bogotá, Colombia: Leyer.

De Colombia, (2006). Código de la infancia y la adolescencia. Bogotá: Colombia.

De Colombia, Ley General Educación, (1994). Ministerio de educación nacional. Bogotá, Colombia.

De Colombia. (1997). Decreto 2247. https://www.mineducacion.gov.co/1621/articles104840 archivo_pdf.pdf Santa Fe de Bogotá, Colombia.

De Colombia. (1959). Declaración de los derechos del niño. Recuperado de https://www.humanium.org/es/declaracion-1959/

De Medellín, (2020) Instituto de Deporte y Recreación, INDER, Medellín.:https://www.inder.gov.co/es/node/44

Dinello, R. (2007). Tratado de educación: propuesta pedagógica del nuevo siglo. Grupo Magro.

(2006). Pedagogía de la Expresión Ludocreativa. Revista Internacional Magisterio, Educación y Pedagogía. Uruguay. (1998) la ludoteca y el circo del pueblo: Reafirmación cultural a través de lo lúdico. En "Juego y Desarrollo Infantil. Un canto a la libertad". Unicef. Bogotá. Colombia. https://docplayer.es/11316005-Juego-y-desarrollo-infantil-un-canto-a-la$\underline{\text { libertad.html }}$

Gardner, H., y Nogués, M. T. M. (1995). Inteligencias múltiples: la teoría en la práctica (Vol. 29). Barcelona: Paidós.

Guardia V. y Kuiyan A. (2017) Juegotecas barriales en la Ciudad de Buenos Aires. Ministerio de Desarrollo Humano y Hábitat, Gobierno de la Ciudad Autónoma de Buenos Aires. Buenos Aires. Argentina.

Honore, C. (2012) Bajo presión. Cómo educar a nuestros hijos en un mundo hiperexigente. RBA Divulgación. 
Lacayo y Coello., (2008). El juego en los niños: enfoque teórico. Revista Educación.

Meneses y Montero (2019) El juego en los niños: enfoque teórico Educación, vol. 25, núm.

2, pp. 113-124 Universidad de Costa Rica San Pedro, Montes de Oca, Costa Rica

Morin, E. (2016). Enseñar a vivir. Manifiesto para cambiar la educación. Barcelona.

España: Espasa Libros

Pavia, V. (2012) El patio escolar: el juego en libertad controlada. Un lugar emblemático.

Territorio de pluralidad. Ed. Novedades Educativas. Buenos Aires. Argentina.

Perrenoud, P. (2007). Desarrollar la práctica reflexiva en el oficio de enseñar. $3^{\text {a }}$. Edición.

Unicef (1989). Convención sobre los derechos del niño.

https://www.unicef.org/panama/spanish/convencion(3).pdf

Solter, A. (2013) Juegos que unen. Cómo solucionar los problemas de comportamiento de $\underline{\text { los niños mediante el juego, la risa y la conexión. Omega. }}$

Valdiño, G. (2015) El juego en la escuela primaria: renovando su presencia y ampliando su sentido. En "Las propuestas de enseñanza y la planificación en la Educación Primaria. Coord. Laura Pitluk. Ed. Homo Sapiens. Rosario. Argentina. 